\title{
Neutrophil-lymphocyte ratio dynamics are useful for distinguishing between recurrence and pseudoprogression in high-grade gliomas
}

This article was published in the following Dove Press journal: Cancer Management and Research

\author{
Yong Huang ${ }^{1} * *$ \\ Haixia Ding ${ }^{1} * *$ \\ Qiuji Wu' \\ Zhiqiang $\mathrm{Li}^{2}$ \\ Huan $\mathrm{Li}^{3}$ \\ Sirui $\mathrm{Li}^{3}$ \\ Conghua $\mathrm{Xie}^{1,4}$ \\ Yahua Zhong'
}

'Department of Radiation and Medical Oncology, Zhongnan Hospital, Wuhan University, Wuhan, Hubei, People's Republic of China; ${ }^{2}$ Department of Neurosurgery, Zhongnan Hospital, Wuhan University, Wuhan, Hubei, People's Republic of China; ${ }^{3}$ Department of Radiology, Zhongnan Hospital, Wuhan University, Wuhan, Hubei, People's Republic of China; ${ }^{4}$ Hubei Cancer Clinical Study Center, Wuhan University, Wuhan, Hubei, People's Republic of China

*These authors contributed equally to this work

\begin{abstract}
Objective: Distinguishing recurrence and pseudoprogression is a major challenge in the clinical practice of treatment for high-grade gliomas (HGGs). The neutrophil-lymphocyte ratio (NLR) has been reported to be closely related to survival in HGGs. We aimed to assess the predictive value of NLR in the differential diagnosis of recurrence and pseudoprogression. Materials and Methods: A total of 135 patients with histologically confirmed HGGs were studied. All patients underwent focal radiotherapy and concomitant temozolomide (TMZ), followed by 6 cycles of TMZ if MRI showed no progressive enlargement of contrastenhancing lesions. MRI evaluation was taken 4 weeks after concurrent chemoradiotherapy and then every 2 months later. NLR was calculated at 4 time points of preoperation, before concurrent RT-TMZ (pretreatment), 4 weeks following completion of RT-TMZ, and MRI showed lesion enlarged or treatment completed.
\end{abstract}

Results: In 135 patients, 47 (34.8\%) were found to be pseudoprogression (PsPD), and 28 (20.7\%) were early disease progression (ePD). The mean pretreatment and post-treatment NLR were $4.2 \pm 2.1$ and $5.1 \pm 3.5$, respectively. The median overall survival in the PsPD group (25.2 months) was significantly longer than in the ePD (15.4 months) and no progression group (nPD) (21.6 months) $(p<0.001)$. Overall survival was significantly shorter in the baseline $\mathrm{NLR} \geq 4$ cohort compared with $\mathrm{NLR}<4 \quad(p=0.03)$, but no significant difference was found between PsPD and ePD $(p=0.197)$. Patients with decreased NLR showed significantly longer survival than no decreased group $(p<0.001)$, and decreased NLR was found to be a significant difference between PsPD and ePD ( $p=0.022)$. Univariate and multivariate logistic regression analyses suggested that decreased NLR was an independent prognosis factor $(p=0.031)$.

Conclusion: Decreased NLR is an independent prognostic factor and is useful for distinguishing between recurrence and pseudoprogression in HGGs.

Keywords: neutrophil-lymphocyte ratio, high-grade gliomas, pseudoprogression, prognostic factors

\section{Introduction}

High-grade gliomas (HGGs) are the most common primary brain malignancy, with an incidence of 3.19 per $100000 .{ }^{1}$ The standard first-line treatment is maximum safe resection followed by radiotherapy with concomitant and adjuvant temozolomide (TMZ). ${ }^{2}$ However, even with a multimodality regimen, the median overall survival is no more than 2 years, and the most common pattern of treatment failue is recurrence. ${ }^{3}$

Pseudoprogression, which is also called therapy-induced injury, is defined as an early radiological increase in the size of contrast-enhancing lesions at magnetic resonance imaging (MRI), and subsequent improvement will occur without any 
further treatment. ${ }^{4}$ The incidence rate of pseudoprogression was $10-40 \%$ in GBM, and it occurs most frequently during the first 3 months after radiation therapy. ${ }^{5-7}$ Although many methods are recommended, including clinical symptoms and brain functional imaging, to distinguish between pseudoprogression and recurrence, it is still very difficult to make a definite conclusion in clinical practice. ${ }^{8}$ It has been suggested that pseudoprogression has significantly better survival than early progression, ${ }^{7}$ so exploring some new methods to help diagnosis is very clinically important.

In multiple solid cancers, an elevated neutrophil-lymphocyte ratio (NLR) has been thought to be related to poorer survival. ${ }^{9-11}$ In patients with GBM, an elevated NLR $>4$ prior to surgery predicted poorer survival. ${ }^{12,13}$ A combined analysis of NLR at baseline and dynamics during treatment was found to be an independent predictor of overall survival in newly diagnosed GBM. ${ }^{14}$

Previous studies have reported the prognostic role of preoperative NLR and NLR dynamics. In this study, we aimed to analyze the value of preoperative NLR and NLR dynamics in the differential diagnosis between recurrence and pseudoprogression in HGGs.

\section{Methods}

\section{Patients}

A total of 135 patients undergoing tumor resection with pathologically confirmed WHO grade III and IV gliomas were included in this study from July 2015 to December 2017. All patients were initially treated without a pre-existing history of gliomas and underwent total or subtotal tumor resection. Patients were treated with postoperative radiotherapy plus continuous daily TMZ (75 mg/ $\mathrm{m} 2 / \mathrm{d}$ ) followed by 6 cycles of maintenance TMZ (150 to $200 \mathrm{mg} / \mathrm{m} 2$ for 5 days every 28 days). MRI was evaluated 4 weeks after concurrent chemoradiotherapy and then every 2 months later.

The study methodologies conformed to the standards set by the Declaration of Helsinki. Approval of the study was obtained from the institutional review board of the Zhongnan Hospital, Wuhan University. All patients who were included in the database signed the informed consent to review and use their medical records.

\section{Imaging evaluation}

Patients were considered to have nonprogressive disease (nPD), and TMZ was continued if MRI presented stable disease or had no evident lesion. Those whose MRI presented lesion growth were classified into two classes: pseudoprogression (PsPD) or early disease progression (ePD). If the subsequent multimodal MRI evaluations (performed after the second or fifth cycle of adjuvant TMZ) showed stable disease or improved, and finally confirmed by multidisciplinary team (MDT) discussion were classified as PsPD, otherwise they were defined as ePD. Treatment was suspended after 6 cycles of TMZ, and TMZ was suspended at anytime if the MRI image evidenced a new lesion outside the radiotherapy field.

\section{Molecular status assessment}

O6-methylguanine-DNA methyltransferase (MGMT) promoter methylation was assessed by methylation-specific PCR, and the isocitrate dehydrogenase 1 (IDH1) mutation was determined by DNA sequencing.

\section{NLR calculation}

Each patient had complete blood counts obtained at preoperative (baseline), before concurrent RT-TMZ (pretreatment), 4 weeks following completion of concurrent RT-TMZ and the time of diognosis as ePD or treatment completely. The NLR was calculated at each time point by dividing the absolute neutrophil count by the absolute lymphocyte count. ${ }^{15}$ The value closest to the time of diognosis as ePD or adjuvant TMZ treatment completely was used for the posttreatment NLR value. The post-treatment NLR minus the NLR at pretreatment is defined as the difference in the NLR $(\triangle N L R)$. Decreased NLR was defined as an NLR decrease more than the standard deviation of the pretreatment NLR, and increased NLR was defined as an NLR increase greater than the standard deviation. The other patients were considered to have a stable NLR.

\section{Statistical analyses}

Statistical significance was set at $p \leq 0.05$. Survival was investigated with the Kaplan-Meier method and log-rank test. Comparisons between the clinicopathological factors and PsPD and ePD were analyzed by Chi-square and Fisher's exact test. Cox proportional hazards modeling was used to investigate survival hazards and binary outcomes.

\section{Results}

Between July 2015 and December 2017, 135 patients with newly diagnosed HGGs were treated with concurrent RT/ TMZ followed by 6 cycles of maintenance chemotherapy 
according to the above-described protocol. The median follow-up of patients included in the analysis was 18 months (range, 5 to 42 months). The patients' baseline characteristics are presented in Table 1.

\section{MRI evaluation}

The first MRI evaluation was performed 1 month after concurrent RT-TMZ, and lesion enlargement was recorded in 63 patients $(46.7 \%)$, while 72 patients were stable. After the second MRI evaluation and MDT discussion, the findings were ePD in $20(31.7 \%)$ of 63 patients and PsPD in $43(68.3 \%)$ of 63 patients. Finally, $47(34.8 \%)$ of 135 patients were found to be PsPD, and 28 (20.7\%) of 135 patients were found to be ePD after the third MRI evaluation and MDT discussion (Figure 1). Among the 28 ePD patients, $16(57.1 \%)$ had taken reoperation and pathology confirmed to be disease progression, and the other 12 patients received 2nd line chemotherapy and faced disease progression in a short period of time.

\section{NLR values}

The mean pretreatment NLR was $4.2 \pm 2.1$, and the mean post-treatment NLR was $5.1 \pm 3.5$. Of 135 patients, 47 patients were assigned to the increased group, 68 patients were assigned to the decreased group, and the other 20 patients were assigned to the stable group. In $47 \mathrm{PsPD}$

Table I Association between clinicopathological variables of patients and PsPD and ePD

\begin{tabular}{|c|c|c|c|c|}
\hline \multirow[t]{2}{*}{ Factors } & \multirow[t]{2}{*}{ All cases No (\%) } & \multicolumn{2}{|c|}{ MRI enlarged } & \multirow[t]{2}{*}{$p$-value } \\
\hline & & PsPD & ePD & \\
\hline Age years & & & & 0.781 \\
\hline$\geq 50$ & 88 & 27 & 17 & \\
\hline$<50$ & 47 & 20 & 11 & \\
\hline Gender & & & & 0.347 \\
\hline Female & $65(48.1)$ & 25 & 18 & \\
\hline Male & $70(51.9)$ & 22 & 10 & \\
\hline WHO performance status & & & & 0.090 \\
\hline 0 & $12(8.9)$ & 8 & 3 & \\
\hline I & $108(80)$ & 35 & 17 & \\
\hline 2 & $15(11.1)$ & 4 & 8 & \\
\hline Extent of surgical resection & & & & 0.301 \\
\hline Subtotal resection & $76(56.3)$ & 28 & 20 & \\
\hline Total resection & $59(43.7)$ & 19 & 8 & \\
\hline MGMT promoter status & & & & $<0.001$ \\
\hline Methylated & $50(37)$ & 38 & 5 & \\
\hline Unmethylated & $85(63)$ & 9 & 23 & \\
\hline IDHI status & & & & 0.535 \\
\hline Wild & $70(51.9)$ & 19 & 15 & \\
\hline Mutant & $28(20.7)$ & 10 & 5 & \\
\hline Unkown & $37(27.4)$ & 18 & 8 & \\
\hline Baseline NLR & & & & 0.197 \\
\hline$\geq 4$ & $65(48.1)$ & 29 & 13 & \\
\hline$<4$ & 70 (5।.9) & 18 & 15 & \\
\hline NLR decreased & & & & 0.022 \\
\hline Decreased & $68(50.4)$ & 28 & 9 & \\
\hline No decreased & $67(49.6)$ & 19 & 19 & \\
\hline NLR increased & & & & 0.128 \\
\hline Increased & $47(34.8)$ & 13 & 13 & \\
\hline No increased & $88(65.2)$ & 32 & 15 & \\
\hline
\end{tabular}




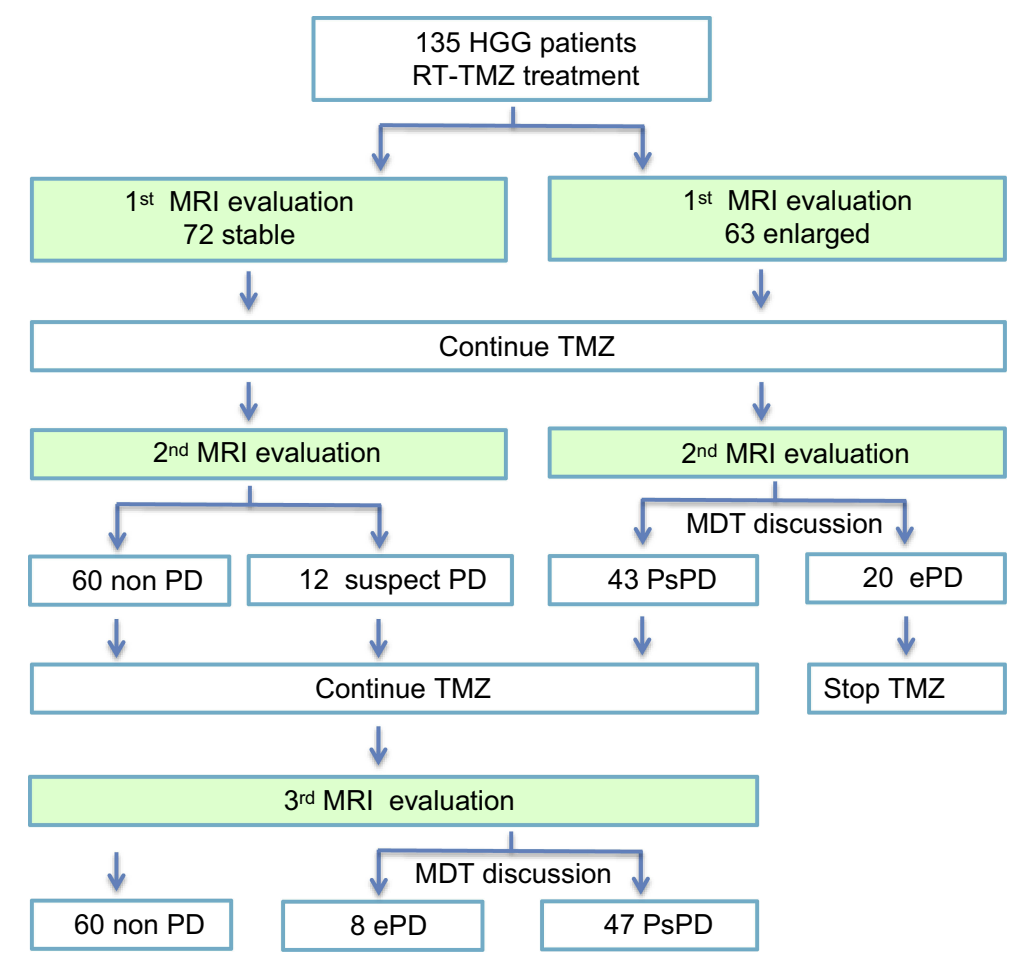

Figure I MRI findings and outcomes of patients.

Abbreviations: MRI, magnetic; resonance imaging; HCGs, high-grade gliomas; TMZ, temozolomide; RT-TMZ, radiotherapy Concurrent TMZ chemotherapy; PD, disease progression; PsPD, pseudoprogression; ePD, early disease progression; MDT, multidisciplinary team.

patients, $28(59.6 \%)$ and $13(27.7 \%)$ were found to be NLR decreased and increased, respectively. Nine $(32.1 \%)$ and $13(46.4 \%)$ in 28 ePD patients were found to be NLR decreased and increased, respectively (Table 1 ).

\section{Survival outcome}

The median overall survival was 22.4 months. In the PsPD group, the median overall survival was 25.2 months, which was significantly longer than in the ePD (15.4 months) and nPD groups $(21.6$ months) $(p<0.001)$ (Figure 2). Overall survival was significantly shorter in the $\mathrm{NLR} \geq 4$ cohort

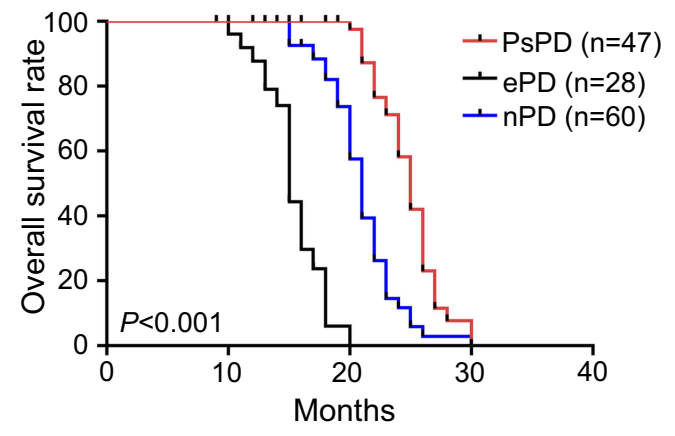

Figure 2 Overall survival for pseudoprogression, early disease progression and neither pseudoprogression nor early disease progression.

Abbreviations: PsPD, pseudoprogression; ePD, early disease progression; nPD, nonprogressive disease. compared with NLR $<4$ ( $p=0.03$ ) (Figure 3 ). Compared with the no-increased group, the increased NLR group showed a trend of decreasing overall survival, but there was no statistically significant difference $(p=0.06)$ (Figure 4). Patients with decreased NLR showed significantly longer survival than the no-decreased group $(p<0.001)$ (Figure 5).

\section{Statistical analysis}

MGMT status $(p<0.001)$ and decreased NLR $(p=0.022)$ were significantly different between PsPD and ePD. No significant difference was found in age $(p=0.781)$, gender

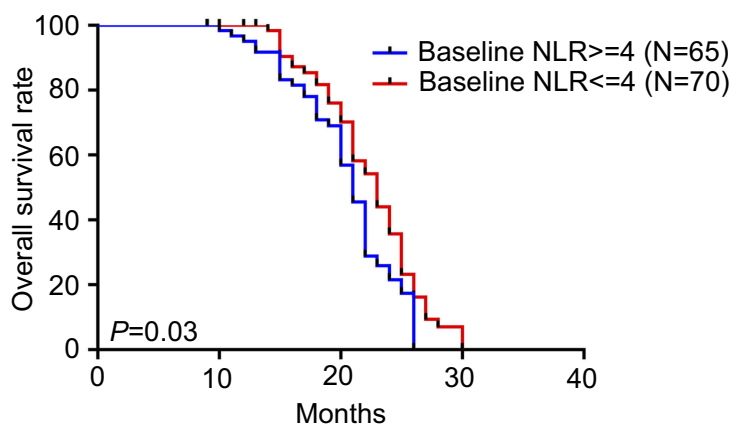

Figure 3 Overall survival for different baseline NLRs.

Abbreviation: NLR, neutrophil-lymphocyte ratio. 


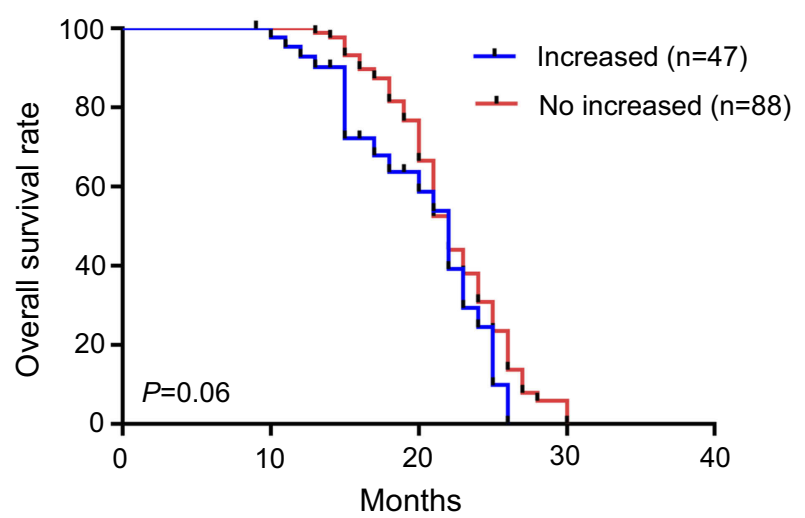

Figure 4 Overall survival for increased and no increased NLR. Abbreviation: NLR, neutrophil-lymphocyte ratio.

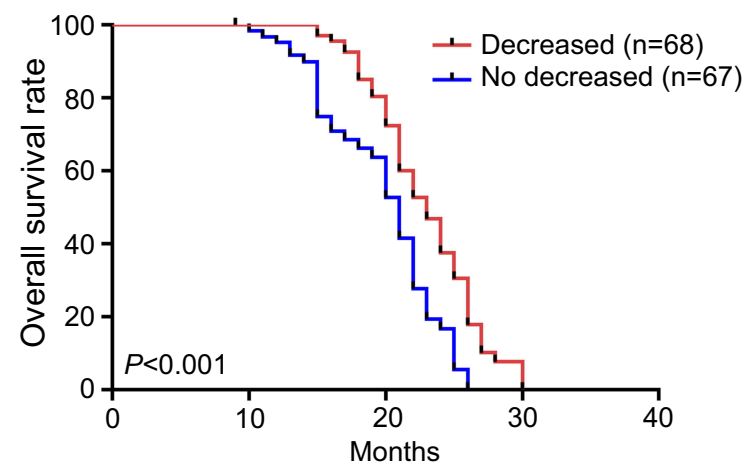

Figure 5 Overall survival for decreased and no decreased NLR. Abbreviation: NLR, neutrophil-lymphocyte ratio.

( $p=0.347)$, WHO performance status $(p=0.090)$, extent of surgical resection $(p=0.301)$, IDH status $(p=0.535)$, baseline NLR $(p=0.197)$ and increased NLR $(p=0.128)$. univariate and multivariate logistic regression analyses were performed to validate whether NLR dynamics were an independent prognostic marker. We found that the extent of surgical resection, MGMT promoter status and decreased NLR are independent prognosis factors (Table 2).

\section{Discussion}

Although pseudoprogression is very common in the treatment of high-grade gliomas, the exact mechanism is still unclear. Tumour-cell and endothelial-cell injury and bloodbrain barrier alteration caused by chemoradiotherapy are thought to be the main process in this event. Unlike disease progression, pseudoprogression is mainly considered to be an adverse event of therapy. ${ }^{16}$ However, even with the application of several modern imaging techniques, such as MRI, MRS, DWI/PWI and PET, correctly and timely distinguishing between pseudoprogression and disease progression is still very difficult. $^{6}$

Previous studies have reported that patients with PsPD have longer OS than those with ePD, but whether the OS of PsPD is longer than nPD remains controversial. ${ }^{7,16}$ In our study, consistent with Brandes et al, we found that PsPD has significantly longer OS than both ePD and nPD.

It is very clear that the tumor-associated immune system plays important roles in tumorigenesis and development. Inflammatory factors, including neutrophils and lymphocytes, have been reported to be closely related to the prognosis and progression of glioblastoma. ${ }^{17}$ Infiltration of immune cells is also suggested to be closely linked with prognosis and IDH status in gliomas. ${ }^{18}$ Previous data also show that tumours can disrupt normal neutrophil homeostasis and increase the number of circulating neutrophils by many pathways. ${ }^{19}$

The neutrophil-lymphocyte ratio (NLR) in peripheral blood, a marker of systemic inflammatory responses, was found to be significantly related to survival and disease stage in many solid tumors. ${ }^{10-12,20-22}$ In non-small cell lung cancer, elevated pre-treatment NLR is suggested to

Table 2 Univariate and multivariate logistic regression analyses for overall survival of high-grade glioma patients

\begin{tabular}{|l|l|l|l|l|l|l|}
\hline \multirow{2}{*}{ Characteristic } & \multicolumn{2}{l|}{ Univariate } & \multicolumn{2}{l|}{ Multivariate } \\
\cline { 2 - 6 } & Odds ratio & $\mathbf{9 5 \%} \mathbf{~ C l}$ & p-value & Odds ratio & $\mathbf{9 5 \%} \mathbf{C l}$ \\
\hline Age & 1.012 & $0.980-1.045$ & 0.475 & & \\
Gender & 0.855 & $0.566-1.293$ & 0.459 & & \\
WHO performance status & 1.312 & $0.808-2.130$ & 0.272 & & \\
Extent of surgery & 0.508 & $0.331-0.778$ & 0.002 & 0.604 & $0.380-0.962$ & 0.034 \\
MGMT promoter status & 0.154 & $0.084-0.281$ & $<0.001$ & 0.160 & $0.085-0.302$ & $<0.001$ \\
IDHI status & 0.629 & $0.466-0.849$ & 0.002 & 0.885 & $0.646-1.212$ & 0.47 \\
Baseline NLR & 1.150 & $1.028-1.286$ & 0.014 & 1.029 & $0.928-1.141$ & 0.584 \\
NLR decreased & 0.474 & $0.306-0.733$ & 0.001 & 0.481 & $0.247-0.934$ & 0.031 \\
NLR increased & 1.584 & $1.064-2.357$ & 0.023 & 0.678 & $0.366-1.254$ & 0.215 \\
\hline
\end{tabular}

Note: Univariate variables with $P<0.2$ were included in the multivariate model.

Abbreviations: IDHI, isocitrate dehydrogenase I; MGMT, O6-methylguanine-DNA methyltransferase; NLR, neutrophil-to-lymphocyte ratio. 
be associated with shorter OS and PFS, and it is an independent predictive factor for patients treated with nivolumab. ${ }^{10}$ After analyzing the relationship between hematological markers and tumor grade, subtype, and clinical outcome, Wang et al found that NLR was the best single hematological marker for predicting tumor grade and survival in patients with gliomas. ${ }^{21}$

In the present study, our findings on outcome confirmed previous studies that patients with preoperative NLR $\geq 4$ have significantly longer survival than those with $\operatorname{NLR}<4^{12,23}$ However, no significant difference was found in the preoperative NLR between PsPD and ePD. Recently, NLR dynamics were also reported to be related to survival in several tumors. Glazer et al found that increased NLR was associated with worse survival in pancreatic ductal adenocarcinoma after neoadjuvant therapy. ${ }^{15}$ Mason et al reported an association between decreased NLR after chemoradiotherapy and longer overall survival. ${ }^{14}$ We also analyzed the difference in NLR dynamics between PsPD and ePD. Unlike baseline NLR, a decreased NLR was not only significantly related to OS but also significantly different between PsPD and ePD. However, no significant difference in increased NLR was found between PsPD and ePD. All these results indicated that decreased NLR has more predictive value in the differential diagnosis of PsPD and ePD than increased NLR. It should be noted that relative neutropenia or lymphocytosis may lead to a decreased NLR, neutropenia was one of the most common side effects caused by chemoradiotherapy, and a decreased NLR may mainly reflect the therapy-induced injury, which was consistent with the definition of PsPD. This may be partly the reason why patients with decreased NLR have better survival and were more likely to be PsPD. It has been confirmed that circulating neutrophil counts were elevated above the normal range in all glioma patients and closely related to tumor grade. ${ }^{24}$ However, in our study, patients with increased NLR had a trend of inferior survival, but the difference between PsPD and ePD patients did not achieve statistical significance, which may indicate that an increased NLR had less predictive value than decreased NLR and that more influencing factors related to increased NLR should be considered in future research.

Recent studies suggest that mutations in IDH1 or IDH2 were associated with longer overall survival than wildtype. ${ }^{25,26}$ IDH mutations were reported to be an independent favorable prognostic marker in HGGs in multivariate analysis. ${ }^{27}$ However, in our study, IDH mutation status was unknown in $27.4 \%$ of patients, which may be the reason why IDH status was not found to be an independent prognostic factor by multivariate logistic regression analyses.
In conclusion, for the first time, we analyzed the predictive value of preoperative NLR and NLR dynamics in the differential diagnosis of PsPD and ePD. The results of our study suggested that decreased NLR is an independent prognostic factor and is useful for distinguishing between recurrence and pseudoprogression for HGGs.

\section{Acknowledgments}

We acknowledge Dr Fang Yu and Dr Qiongrong Chen for help with DNA sequencing. This work was surpported by the National Natural Science Foundation of China (81641116) and Health and Family Planning Commission Foundation of Hubei Province (WJ2017H0007).

\section{Disclosure}

The authors report no conflicts of interest in this work.

\section{References}

1. Ostrom QT, Gittleman H, Farah P, et al. CBTRUS statistical report: Primary brain and central nervous system tumors diagnosed in the United States in 2006-2010. Neuro Oncol. 2013;15:ii1-ii56. doi:10.1093/neuonc/not151

2. Alexander BM 1, Cloughesy TF. Adult glioblastoma. J Clin Oncol. 2017;35(21):2402-2409. doi:10.1200/JCO.2017.73.0119

3. Linz U. Commentary on effects of radiotherapy with concomitant and adjuvant temozolomide versus radiotherapy alone on survival in glioblastoma in a randomised phase III study: 5-year analysis of the EORTC-NCIC trial (lancet oncol. 2009;10:459-466). Cancer. 2010;116(8):1844-1846. doi:10.1002/cncr.24950

4. Wen PY, Macdonald DR, Reardon DA, et al. Updated response assessment criteria for high-grade gliomas: response assessment in neuro-oncology working group. J Clin Oncol. 2010;28(11):1963-1972. doi:10.1200/JCO.2009.26.3541

5. Brandsma D, Stalpers L, Taal W, Sminia P, van Den Bent MJ. Clinical features, mechanisms, and management of pseudoprogression in malignant gliomas. Lancet Oncol. 2008;9:453-461. doi:10.1016/S1470-2045(08)70125-6

6. Thust SC, van den Bent MJ, Smits M. Pseudoprogression of brain tumors. J Magn Reson Imaging. 2018;48(3):571-589. doi:10.1002/jmri.26171

7. Brandes AA, Franceschi E, Tosoni A, et al. MGMT promoter methylation status can predict the incidence and outcome of pseudoprogression after concomitant radiochemotherapy in newly diagnosed glioblastoma patients. J Clin Oncol. 2008;26(13):2192-2197. doi:10.1200/JCO.2007.14.8163

8. Chiang GC, Kovanlikaya I, Choi C, Ramakrishna R, Magge R, Shungu DC. Magnetic resonance spectroscopy, positron emission tomography and radiogenomics-relevance to glioma. Front Neurol. 2018;5(9):33. doi:10.3389/fneur.2018.00033

9. Hata T, Mizuma M, Motoi F, et al. Diagnostic and prognostic impact of neutrophil-to-lymphocyte ratio for intraductal papillary mucinous neoplasms of the pancreas with high-grade dysplasia and associated invasive carcinoma. Pancreas. 2019;48(1):99-106. doi:10.1097/ MPA.0000000000001202

10. Diem S, Schmid S, Krapf M, et al. Neutrophil-to-Lymphocyte ratio (NLR) and Platelet-to-Lymphocyte ratio (PLR) as prognostic markers in patients with non-small cell lung cancer (NSCLC) treated with nivolumab. Lung Cancer. 2017;111:176-181. doi:10.1016/j. lungcan.2017.07.024 
11. Jonska-Gmyrek J, Gmyrek L, Zolciak-Siwinska A, et al. Pretreatment neutrophil to lymphocyte and platelet to lymphocyte ratios as predictive factors for the survival of cervical adenocarcinoma patients. Cancer Manag Res. 2018;10:6029-6038. doi:10.2147/CMAR.S178745

12. Templeton AJ, McNamara MG, Seruga B, et al. Prognostic role of neutrophil-to-lymphocyte ratio in solid tumors: a systematic review and meta-analysis. J Natl Cancer Inst. 2014;106:dju124. doi:10.1093/ jnci/dju061

13. Zhang J, Zhang S, Song Y, et al. Prognostic role of neutrophil lymphocyte ratio in patients with glioma. Oncotarget. 2017;8 (35):59217-59224. doi:10.18632/oncotarget.19484

14. Mason M, Maurice C, McNamara MG, et al. Neutrophil-lymphocyte ratio dynamics during concurrent chemo-radiotherapy for glioblastoma is an independent predictor for overall survival. $J$ Neurooncol. 2017;132(3):463-471. doi:10.1007/s11060-017-2395-y

15. Glazer ES, Rashid OM, Pimiento JM, et al. Increased neutrophil-tolymphocyte ratio after neoadjuvant therapy is associated with worse survival after resection of borderline resectable pancreatic ductal adenocarcinoma. Surgery. 2016;160(5):1288-1293. doi:10.1016/j. surg.2016.04.039

16. Balaña C, Capellades J, Pineda E, et al. Pseudoprogression as an adverse event of glioblastoma therapy. Cancer Med. 2017;6 (12):2858-2866. doi:10.1002/cam4.1242

17. Han S, Liu Y, Li Q, et al. Pre-treatment neutrophil-to-lymphocyte ratio is associated with neutrophil and T-cell infiltration and predicts clinical outcome in patients with glioblastoma. BMC Cancer. 2015;15:617. doi:10.1186/s12885-015-1584-3

18. Amankulor NM, Kim Y, Arora S, et al. Mutant IDH1 regulates the tumorassociated immune system in gliomas. Genes Dev. 2017;31 (8):774-786. doi:10.1101/gad.294991.116

19. Coffelt SB, Wellenstein MD, de Visser KE. Neutrophils in cancer: neutral no more. Nat Rev Cancer. 2016;16(7):431-446. doi:10.1038/ nrc. 2016.52
20. McNamara MG, Lwin Z, Jiang H, et al. Factors impacting survival following second surgery in patients with glioblastoma in the temozolomide treatment era, incorporating neutrophil/lymphocyte ratio and time to first progression. J Neurooncol. 2014;117(1):147-152. doi:10.1007/s11060-014-1366-9

21. Wang PF, Meng Z, Song HW, et al. Preoperative changes in hematological markers and predictors of glioma grade and survival. Front Pharmacol. 2018;9:886. doi:10.3389/fphar.2018.00886

22. Liang J, Piao Y, Holmes L, et al. promote the malignant glioma phenotype through S100A4. Clin Cancer Res. 2014;20(1):187-198. doi:10.1158/1078-0432.CCR-13-1279

23. Bambury RM, Teo MY, Power DG, et al. The association of pre-treatment neutrophil to lymphocyte ratio with overall survival in patients with glioblastoma multiforme. J Neurooncol. 2013;114 (1):149-154. doi:10.1007/s11060-013-1164-9

24. Fossati G, Ricevuti G, Edwards SW, Walker C, Dalton A, Rossi ML. Neutrophil infiltration into human gliomas. Acta Neuropathol. 1999;98(4):349-354. doi:10.1007/s004010051093

25. Hartmann C 1, Hentschel B, Wick W, et al. Patients with IDH1 wild type anaplastic astrocytomas exhibit worse prognosis than IDH1-mutated glioblastomas, and IDH1 mutation status accounts for the unfavorable prognostic effect of higher age: implications for classification of gliomas. Acta Neuropathol. 2010;120(6):707-718. doi:10.1007/s00401-010-0781-z

26. Nobusawa S, Watanabe T, Kleihues P, et al. IDH1 mutations as molecular signature and predictive factor of secondary glioblastomas. Clin Cancer Res. 2009;15(19):6002-6007. doi:10.1158/1078-0432.CCR-09-0715

27. Ichimura K, Pearson DM, Kocialkowski S, et al. IDH1 mutations are present in the majority of common adult gliomas but rare in primary glioblastomas. Neuro Oncol. 2009;11(4):341-347. doi:10.1215/ 15228517-2009-025
Cancer Management and Research

\section{Publish your work in this journal}

Cancer Management and Research is an international, peer-reviewed open access journal focusing on cancer research and the optimal use of preventative and integrated treatment interventions to achieve improved outcomes, enhanced survival and quality of life for the cancer patient.
The manuscript management system is completely online and includes a very quick and fair peer-review system, which is all easy to use. Visit http://www.dovepress.com/testimonials.php to read real quotes from published authors. 